\title{
Analysis of the effects of uneven sound coverage over a facade during a sound insulation test according to the international standard ISO 16283-3
}

\author{
C. Asensio , J.A. Trujillo, G. Arcas
}

\begin{abstract}
A B S T R A C T
Building regulations worldwide include specific sections on protection against noise and vibrations, making sound insulation of facades an important part of regulations in many countries. Consequently, sound insulation field tests become important, as a way to certify, or check, if a facade complies with the regulatory requirements. The international standard ISO 16283-3:2016 specifies the procedures and methods to describe the sound insulation of a facade, making the loudspeaker method for the whole facade one of the most widely used because of the advantages it has for practitioners.

To make the method consistent, the international standard establishes some constraints, trying to minimize the variation of sound pressure level on the facade. This variation is caused by the directivity of the loudspeaker and the distance from the loudspeaker to the facade, causing an uneven sound coverage over the facade. This paper analyzes and quantifies the solitary influence of facade coverage on the sound insulation determinations in relation to the location of the facade elements. In addition to other influences, the specific location of a small air inlet on a facade can alter the results by up to $4 \mathrm{~dB}$, even if the sound coverage complies with the requirements established by the international standard. Finally, an alternative measurement setup is discussed that aims to minimize the negative effect of this phenomenon in an attempt to increase the consistency of the method.
\end{abstract}

\section{Introduction}

The continuous increase in community noise in cities, together with the higher concern of citizens, continuously causes increasing attention to be paid to the performance of buildings at protecting from outdoor noise [1]. Building regulations in many countries include specific sections on protection against noise and vibrations, making sound insulation of facades an important part of the facades [2-4]. Since the regulation requires a degree of protection against noise, field measurements become important, as a way to certify, or check, if a building complies with the regulatory requirements.

Facade sound insulation tests are intended to describe the noise reduction that a facade produces in the presence of outdoor noise. The International Standard ISO 16283-3:2016 [5] specifies procedures to determine the airborne sound insulation of facade elements (element methods) and whole facades (global methods) using sound pressure measurements. The test method mainly implies the measurement of outdoor and indoor noise levels that a specific outdoor noise source produces. Depending on the noise source to be used (aircraft traffic, road traffic or loudspeaker), and if the whole facade or an element is to be described, a slightly different measurement method must be applied.

This research focuses on the loudspeaker global method (for the whole facade). ISO $16283-3$ specifies the measurement setup as follows (basic description):

- The loudspeaker position and the distance to the facade must minimize the variation of the sound pressure level on the facade. The distance from the loudspeaker to the center of the facade must be at least $7 \mathrm{~m}$, and the angle of the sound incidence must be $45^{\circ} \pm 5^{\circ}$.

- The reference microphone position for outdoor measurement $\left(\mathrm{L}_{1,2 \mathrm{~m}}\right)$ is in front of the center of the facade, at a distance $2 \pm 0.2 \mathrm{~m}$ from the plane of the facade.

The loudspeaker method has a number of advantages for practitioners, mainly because they can have full control over the noise source and are able to determine its location, color, initiation and strength. Loudspeaker-based tests are easier to perform, and the determinations are less corrupted by the background noise, as 
the practitioner will always have the ability to turn up the volume of the source.

However, the use of loudspeakers has a main drawback, which is derived from the non-uniform sound pressure level on each part of the facade. To minimize this effect, ISO 16283-3 establishes some constraints on the loudspeaker's emissions: "The directivity of the loudspeaker in the free field shall be such that the local differences in the sound pressure level in each frequency band of interest are less than $5 \mathrm{~dB}$, as measured on an imaginary surface of the same size and orientation as the test specimen". The directivity of the loudspeaker, and the distance from the loudspeaker to any part of the facade, may cause an uneven sound coverage beyond the constraints in this standard, especially when there is not much free space in front of the facade and it is not possible to locate the speaker farther away.

These constraints have been studied in depth by Professor Sánchez and colleagues, from the Universidad Politécnica de Madrid [6-10]. They have analyzed the influence that the geometry of a facade and the directivity of the source have on the incident sound level during a test to find the maximum size of a facade that meets the requirements in the standard (ISO 140-5 at that time) for the typical radiation pattern of commercial loudspeakers. They have also proposed a laboratory procedure for verification of sound source coverage over facades according to the International Standard ISO 140-5 [11] that can also be applied for the revised ISO 16283-3.

In addition to the specifications that the loudspeakers must comply with (coverage), sound reduction determinations might vary depending on the sound intensity incident to each of the elements of a facade, which is determined by the location of the loudspeaker and its directivity pattern. Furthermore, for the same measurement setup, different results can be observed depending on the location of the facade's elements (Fig. 1).

The methodology defined in this research has three objectives: (a) to analyze the influence that the directivity of the source and the location of the elements can have on the facade sound insulation measurement results, (b) to quantify this phenomenon and its contribution to the variability/consistency of the measurement method, and (c) to discuss some optimization options that could be addressed in future updates of the international standard.

\section{Methodology}

In this study, two different models describe the acoustics during a sound insulation test according to the loudspeaker method. A loudspeaker in front of the facade emits wideband noise with an incident angle of $45^{\circ}$ (Fig. 2). The first model is used to predict the sound intensity distribution along the external facade's face $\left(L_{1}\right)$. The second model is used to calculate the sound intensity distribution along the internal face of the facade $\left(L_{2}\right)$. The difference between both results $\left(\mathrm{L}_{1}\right.$ and $\left.\mathrm{L}_{2}\right)$ determines the sound insulation of the facade for a given facade and measurement setup. This method was applied to different scenarios to study the influence of the directivity and the location of the facade elements on the test results.

\subsection{The outdoor noise model}

The sound intensity on every piece of facade is not uniform, as it depends on (a) the distance from the source, and (b) the loudspeaker's directivity (angle $\alpha$ ).

To predict the incident sound intensity along the facade, the facade was split into small parts. Then, the propagation of the sound outdoors was calculated using the main elements mentioned in ISO 9613 [12], and the model represented by Eq. (1) is used to predict the sound intensity at every small discrete section making up the facade.

$L_{1}=L_{\text {ref }}-20 \log r-D I(\alpha)-A_{\text {other }}$

where

$\mathrm{L}_{1}$ stands for the incident sound intensity level at a given piece of the facade,

$\mathrm{L}_{\mathrm{ref}}$ is a constant that depends on the strength of the source and does not change during the tests,

$r$ is the distance from the source to the specific piece of facade under analysis,

$\operatorname{DI}(\alpha)$ stands for the directivity index in the direction of the element (angle $\alpha$ ), and

$A_{\text {other }}$ stands for all other effects, which will remain fixed during this analysis (ground reflection, atmospheric attenuation...).

This research focuses only on the effect of uneven sound coverage, as its influence does not depend on other factors, and the conclusions can be generalized. Therefore, other effects that might affect the non-uniformity of the incident sound field in certain cases (i.e., ground reflections, interferences) have been rejected.

\subsection{The sound insulation model}

The sound reduction index $\left(\mathrm{R}^{\prime}\right)$ describes the performance of the facade, and "it hypothetically can be measured with a diffuse incident sound field in the actual field situation" [13]. This descriptor is the reference value in this research, as it can be used to derive any other descriptors. The loudspeaker characteristics are not a limita-

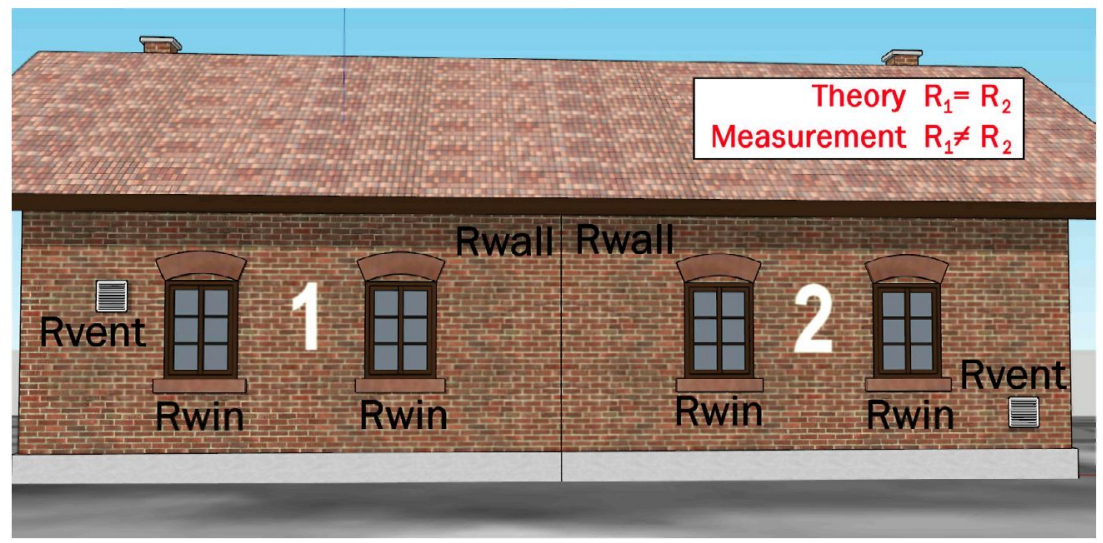

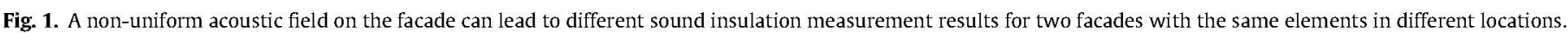




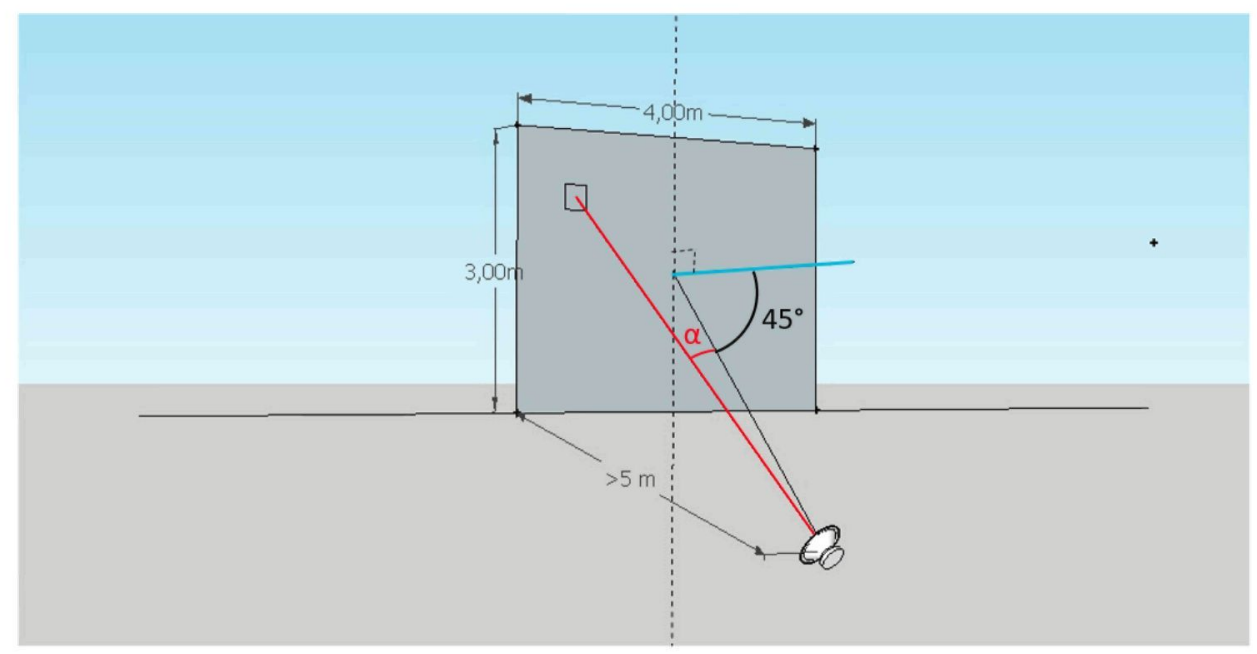

Fig. 2. A non-uniform acoustic field on a facade can lead to different sound insulation measurement results for two facades with the same elements.

tion in our "virtual tests", and it is possible to simulate tests using a loudspeaker that can provide a uniform incident sound all over the facade. For simplicity, the effect of the coincidence frequency has not been considered, as it is outside the scope of this research, and the correction to be applied does not depend on the uneven sound coverage. According to this assumption, the relation in Eq. (2) is derived.

$R_{45^{\circ}}^{\prime}=R^{\prime}$

According to ISO 12354-3 [13], the reference value $\left(\mathrm{R}^{\prime}\right)$ in this research can be obtained using Eq. (3), as flanking transmissions can be neglected (uneven coverage could also have an effect on flanking transmissions, but flanking transmissions are not being focused on in this research; therefore, it is outside the scope of this paper).

$R^{\prime}=-10^{*} \log \left(\sum_{i=1}^{n} \tau_{e, i}\right)$

where

$\tau_{e, i}$ is the sound power ratio of the radiated sound power by a facade element $i$ due to the direct transmission of incident sound on this element, relative to the incident sound power on the total facade. It can be obtained according to Eq. (4) for small technical elements, or Eq. (5) for other elements.

$\tau_{e, i}=\frac{A_{0}}{S} \cdot 10^{-0,1 D_{n, i}}$

$\tau_{e, i}=\frac{S_{i}}{S} \cdot 10^{-0,1 R_{i}}$

where,

$R_{i}$ is the sound reduction index in decibels of the element $i$,

$S_{i}$ is the area of element $i$, in square meters,

$S$ is the total area of the facade as seen from the inside, in square meters,

$A_{0}$ is $10 \mathrm{~m}^{2}$, and

$D_{n, e, i}$ is the element of the normalized sound level difference in decibels of the small element $i$.

It has been considered that the reverberation time, or the absorption of the room does not change when the facade elements move from one location to another. Moreover, each scenario maintains the source location and its directivity as a constant. Consequently, the sound level outdoors does not change for different iterations (the sound pressure level at any given location outdoors stays the same for each iteration), and the variability in the results (each scenario) is only derived from the specific location of the facade elements (windows, ventilation...). For this reason, even if other descriptors were analyzed, or other relations between $R_{45^{\circ}}$ and $R^{\prime}$ were assumed (ISO 12354-3 suggests $R_{45^{\circ}}^{\prime}=R^{\prime}+1$, in average), the exact same conclusions would be obtained (same variability, biased results).

When applying ISO12354-3 to estimate $\mathrm{R}^{\prime}$, only the sound reduction index of the facade elements and their sizes are considered. The directivity of the source and the specific location of the facade's elements are not considered; therefore, the obtained value will be used as a reference to study the effect of these new factors. This research sets the focus on the non-uniform incident sound level; therefore, the sound intensity at every piece of façade has been calculated first, according to Eq. (1). Then, depending on whether piece $i$ is a part of a small technical element or any other element, Eq. (4) or Eq. (5) is used to estimate the attenuation of sound. As an example, Eq. (6) shows the formula used to calculate the sound reduction index of the facade, in the case of a window. In the case of a uniform incident sound distribution along the wall, Eqs. (6) and (3) would give the same results (this is also true if all the facade elements have the same sound reduction indexes).

$R_{45^{\circ}}^{\prime}=10 \cdot \log \left(\sum_{i=1}^{n} 10^{0.1 L_{1 i}}\right)-10 \cdot \log \left(\sum_{i=1}^{n} 10^{0.1\left(L_{1 i}-R_{i}\right)}\right) \quad i \in[1 \ldots n]$

The variable $R_{i}$ is the sound reduction index of the window, and $\mathrm{n}$ is the number of equivalently sized pieces into which the facade was split.

Of course, this calculation must be applied to every frequency band being analyzed, but for clarity of the explanations, only one of them (any of them) is presented. Fig. 3 shows the distribution of the sound intensity in a given third octave band $(200 \mathrm{~Hz})$ along the facade, while Fig. 4 shows the transmitted sound.

\section{Results}

The described methodology has been applied to different scenarios to calculate the difference between the reference sound reduction index $\left(\mathrm{R}^{\prime}\right)$ and the test-based calculated sound reduction index $\left(\mathrm{R}_{45}^{\prime}\right)$ in order to analyze two different factors: the loudspeaker directivity and the location of the weakest element. 


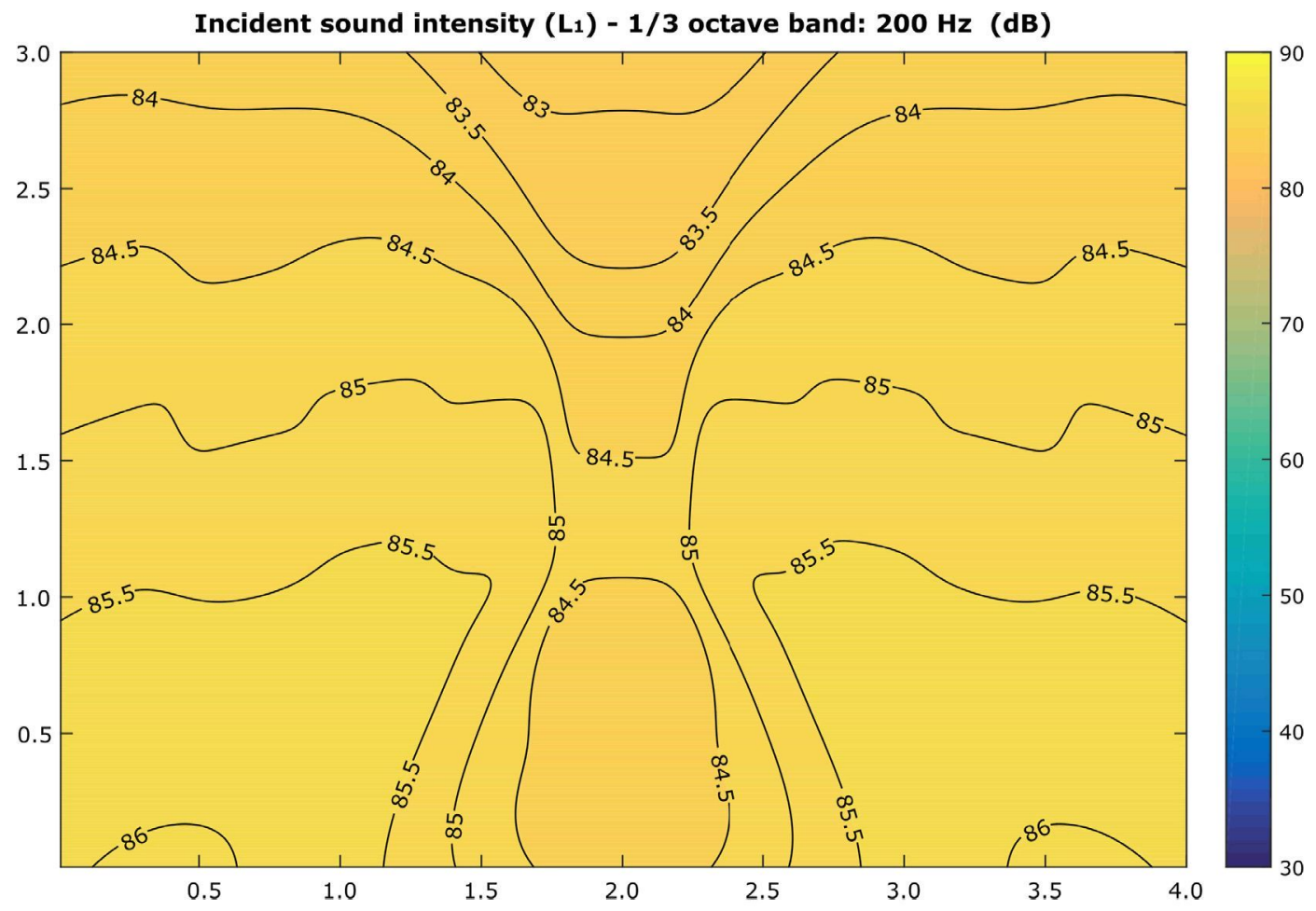

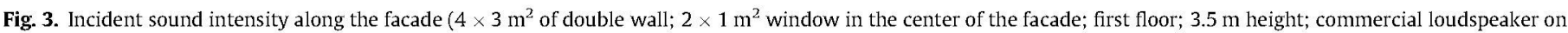
the ground, distance to the facade is $5 \mathrm{~m}$ ).

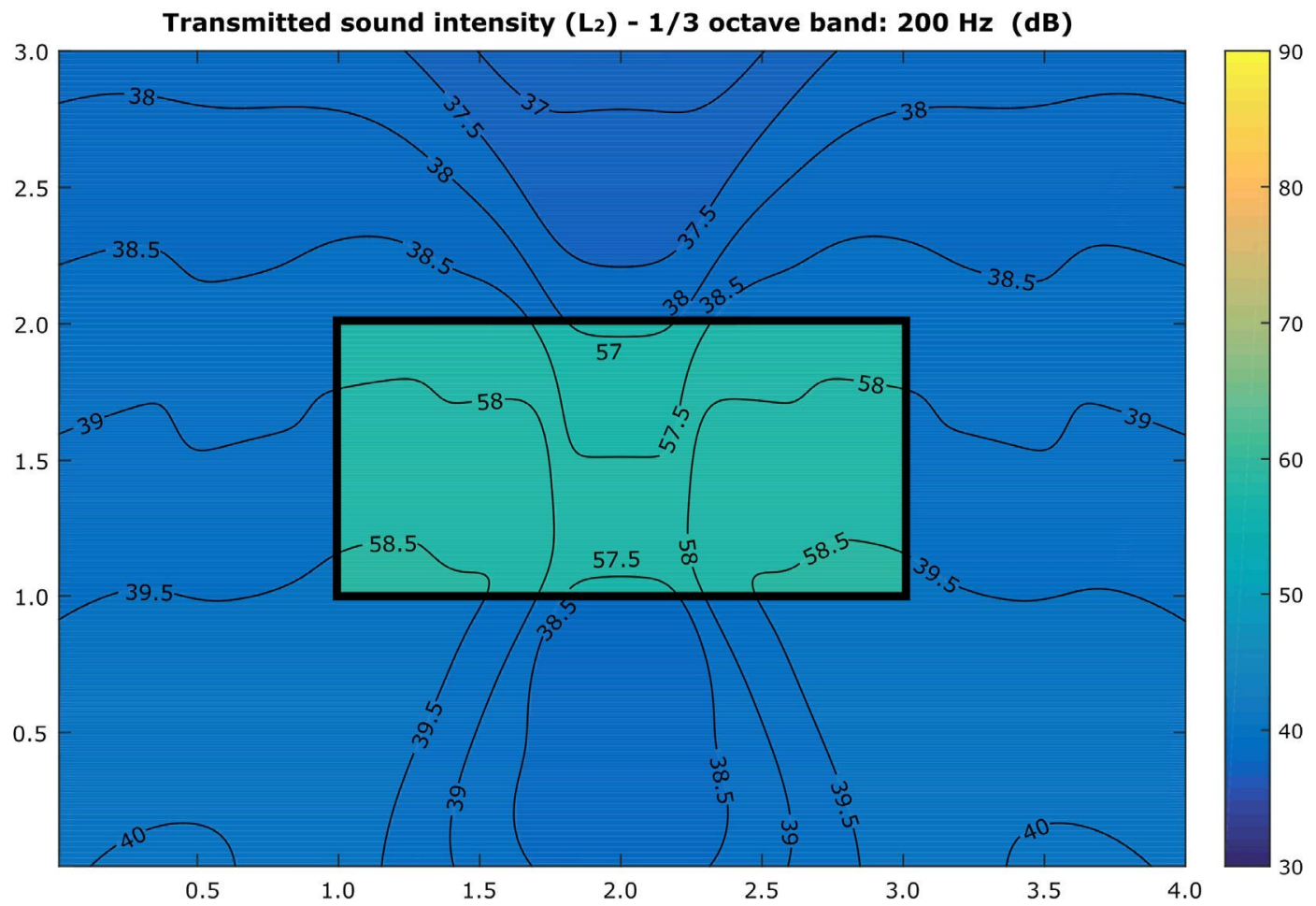

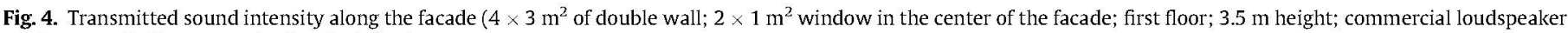
on the ground, distance to the facade is $5 \mathrm{~m}$ ).

\subsection{The effect of the facade elements' locations}

This section analyzes how the measured sound reduction index changes, depending on the position of the weakest facade element. The analyses have been performed under some common conditions:
- A $4 \times 3 \mathrm{~m}^{2}$ double wall facade, which is the size that Sanchez et al. used in [6].

- Just one element (a window in Scenario 1 and an air inlet in Scenario 2 ) in the facade was selected as a higher influence was expected for the ratio between the strongest and weakest elements in the facade. 
- The facade is on the first floor, $3.5 \mathrm{~m}$ over the ground. This setup was selected to minimize the distance-effect on the variation of the incident sound [6].

- A loudspeaker on the ground was used with a distance of $5.0 \mathrm{~m}$ from the facade and $7.0 \mathrm{~m}$ from the facade center for a $45^{\circ}$ incident sound pointing to the facade center. The methodology can be applied to any other setup, but this location was selected to fulfill the minimum requirements in the international standard.

- Only results in the 125 and $5000 \mathrm{~Hz}$ frequency bands are presented, as these are representative of the low and high frequency ranges.

The sound source was selected to strictly meet the criterion that the difference between the lowest and the highest incident sound level in the facade is $5 \mathrm{~dB}$, which is the most unfavorable case that meets the ISO 16283-3 requirements. For simplicity, a piston mounted in an infinite baffle has been used with its size adjusted (radius $=0.06 \mathrm{~m}$ ) for its main directivity lobe to meet this criterion in the $5 \mathrm{kHz}$ third-octave frequency band (Fig. 5). This incident sound field remains constant in scenarios 1 and 2 .

Table 1 shows the sound reduction index of the facade elements; these values have been taken from the annexes in ISO 12354-3 in octave bands, and have been assumed for the third octave band calculations, but any other values could have been chosen.

\subsubsection{Scenario 1. Moveable window with a $6-\mathrm{mm}$ pane}

In this scenario, the double wall facade has only one element, which is a $1 \mathrm{~m} \times 1 \mathrm{~m}$ window with a $6-\mathrm{mm}$ pane. The sound insulation results have been simulated for all locations of the window along the wall (keeping a $0.1-\mathrm{m}$ margin at the edges), using $0.2-\mathrm{m}$ intervals (Fig. 6).

Depending on the window's location, the calculated sound insulation will be different, and variability arises. Fig. 7 maps the results for the 125 and $5000 \mathrm{~Hz}$ third octave bands for every loca- tion of the window. In the low frequency range, the directivity of the loudspeaker is quite low; therefore, the source-receiver distance is the main factor responsible for the non-uniformity of the incident sound. The sound intensity does not change much along the wall, and there is little influence from the window location When the window is located on the top part of the facade, the sound reduction index is overestimated, while when the window is on the bottom part, the results are underestimated. The results vary in an approximate $\pm 0.5-\mathrm{dB}$ interval, depending on the location of the window. The mean value of this series of results is not biased, and its variability is very small (mean $_{125}=0.00 \mathrm{~dB}$, $\operatorname{std}_{125}=0.13 \mathrm{~dB}$ ). The situation in the high frequency range is significantly different as the non-uniformity is mainly caused by the directivity of the source. When the window is placed in the lower central part of the facade or the window is closer to the top corners of the facade, the insulation can be underestimated by more than $1.5 \mathrm{~dB}$. When the window is placed in the center of the facade, the insulation is also underestimated by up to $1 \mathrm{~dB}$; as in this case, the influence of the window's location is not negligible for both the vertical and horizontal axes (mean $_{5000}=-0.50 \mathrm{~dB}$ $\left.\operatorname{std}_{5000}=0.64 \mathrm{~dB}\right)$.

of course, the size of the window will affect the sound insulation of the whole facade, but beyond the area of the element, this research aimed to analyze the interaction between the nonuniform sound intensity, the size, shape and location of the window, and the effect on the field sound reduction index. The previous experiment has been repeated for different window sizes (from $0.2 \times 0.2$ to $2 \times 2$ ). Fig. 8 shows the results in a scatter plot, where each window size that was tested has 4 results $\left(\operatorname{mean}_{125}\right.$, mean $_{5000}$, $\operatorname{std}_{125}$ and $\mathrm{std}_{5000}$ ).

In the low frequency range, the location of the window produces a small variability in the method and a negligible bias. The standard deviation $\left(\mathrm{std}_{125}\right)$ is approximately $0.5 \mathrm{~dB}$ for an approximate $1 \mathrm{~m}^{2}$ window size, and it is smaller when the window is bigger.

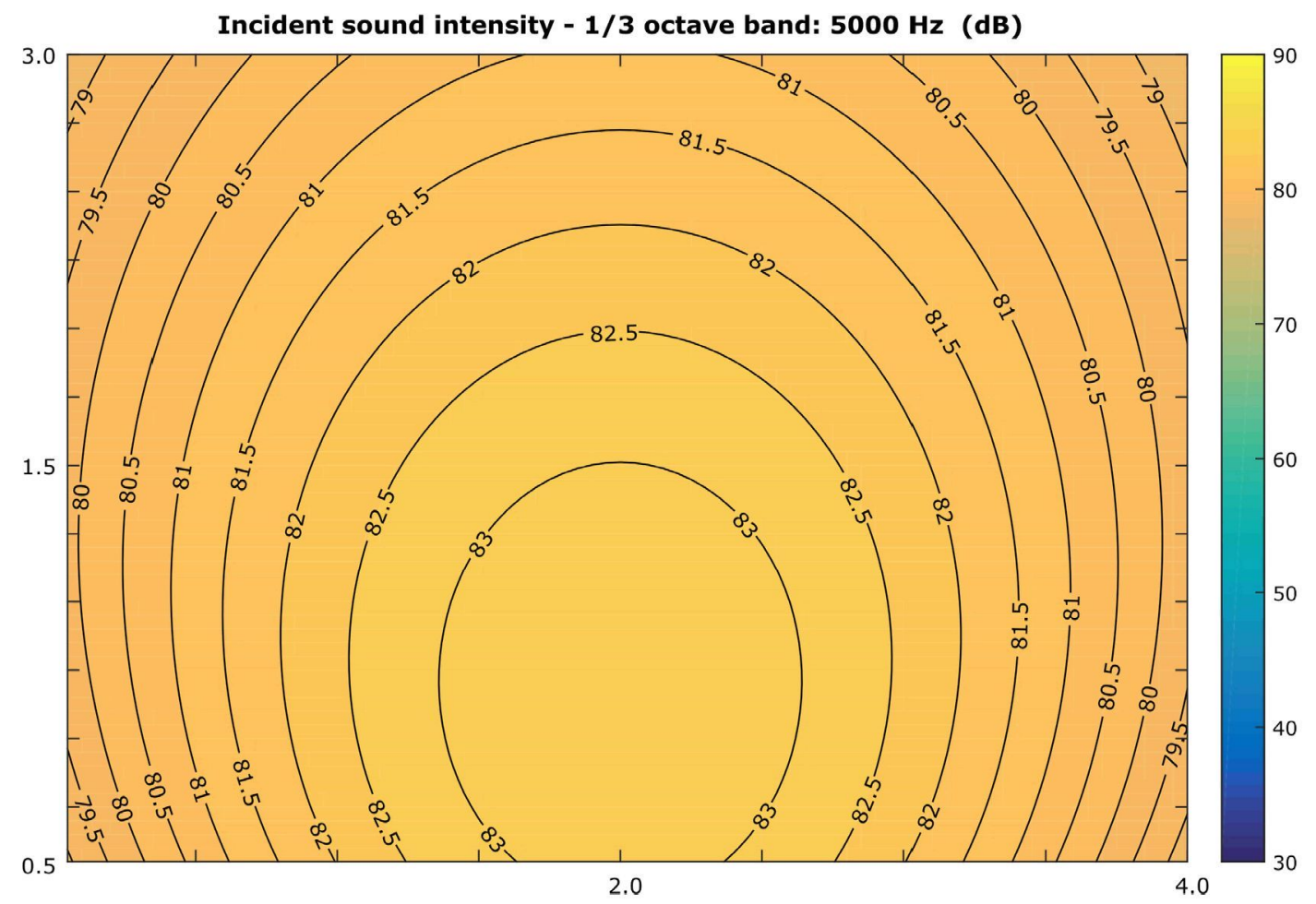

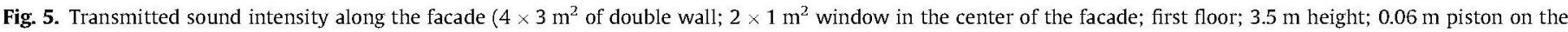
ground, distance to the facade is $5 \mathrm{~m}$ ) 
Table 1

Sound reduction index of the facade elements.

\begin{tabular}{|c|c|c|c|c|c|c|}
\hline \multirow[t]{2}{*}{ Element } & \multicolumn{6}{|c|}{ Sound reduction index $(\mathrm{dB})$} \\
\hline & $125 \mathrm{~Hz}$ & $250 \mathrm{~Hz}$ & $500 \mathrm{~Hz}$ & $1000 \mathrm{~Hz}$ & $2000 \mathrm{~Hz}$ & $4000 \mathrm{~Hz}$ \\
\hline Double wall, $400 \mathrm{~kg} / \mathrm{m}^{2}$ (both scenarios) & 41 & 46 & 52 & 58 & 64 & 70 \\
\hline Window, $6 \mathrm{~mm}$ (Scenario 1) & 24 & 27 & 30 & 33 & 30 & 35 \\
\hline Air inlet (Scenario 2) & 0 & 0 & 0 & 0 & 0 & 0 \\
\hline
\end{tabular}

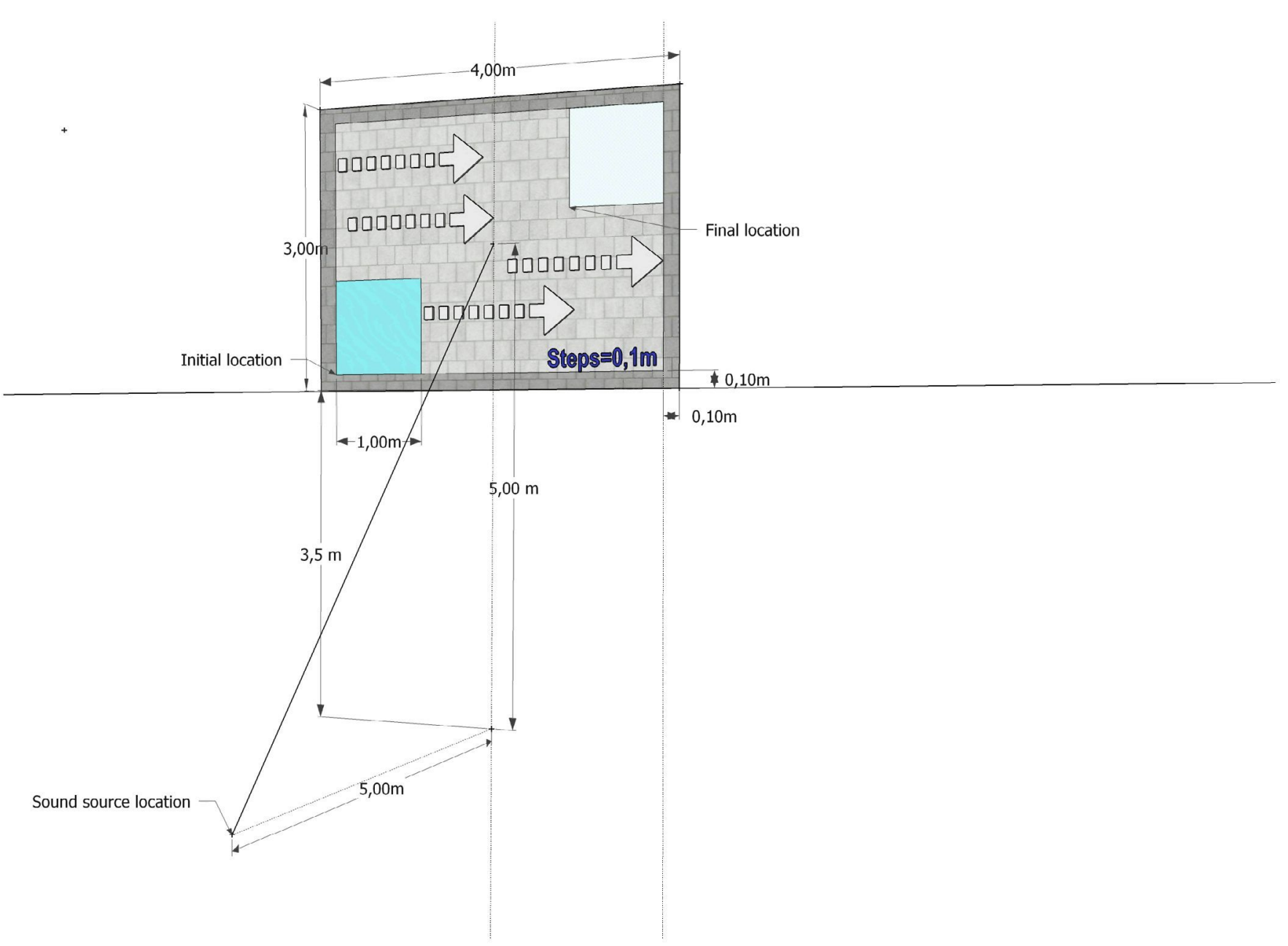

Fig. 6. Scenario 1 setup ( $4 \times 3 \mathrm{~m}^{2}$ of double wall; $1 \times 1 \mathrm{~m}^{2}$ window, placed along the facade in $0.1-\mathrm{m}$ steps).

When the directivity of the loudspeaker is larger (high frequency), a negative bias appears, which is related to when the window's location is closer to the loudspeaker axis. The variability of the observations $\left(s t d_{5000}\right)$ is much higher in the high frequency range, but it decreases as the window size increases.

It has been also observed that the shape of the window affects the results when the directivity is high (Fig. 9). For a fixed window area ( $1 \mathrm{~m}^{2}$ in Fig. 9 ), the bias increases with the horizontal dimension of the element, while the standard deviation increases with the vertical dimension. However, in both cases, the effect is very limited.

\subsubsection{Scenario 2. Moveable air inlet}

In this scenario, the facade does not have a window but instead has a $0.2 \times 0.2 \mathrm{~m}^{2}$ air inlet. The element on the wall is significantly smaller than in scenario 1 , but its sound insulation is smaller. Consequentially, both the low and high frequency bands show a higher sensitivity to the element's location. While in scenario 1 , the results ranged around approximately $2.5 \mathrm{~dB}(5000 \mathrm{~Hz}$ band), depending on the window location, in scenario 2 , the range increases up to $4.0 \mathrm{~dB}$ (Fig. 10). Consequently, the variability of the method is higher than that in scenario 1 :

$\operatorname{mean}_{125}=0.04 \mathrm{~dB} ; \quad \operatorname{mean}_{5000}=-0.07 \mathrm{~dB} ; \quad \operatorname{std}_{125}=0.65 \mathrm{~dB} ;$

$\operatorname{std}_{5000}=1.01 \mathrm{~dB}$

Fig. 11 shows the results as a function of the air inlet size. When Fig. 8 (window) and 11 (air inlet) are compared, the following conclusions can be drawn:

- The bias mainly depends on the frequency range (this is related to directivity) and the size of the element, but it does not change significantly when the window and the air inlet are interchanged.

- The variability in the $5000 \mathrm{~Hz}$ band does not change significantly when the window is replaced by an air inlet of the same size, meaning that in both cases, the sound reduction index of the element is significantly lower than the wall's value. 

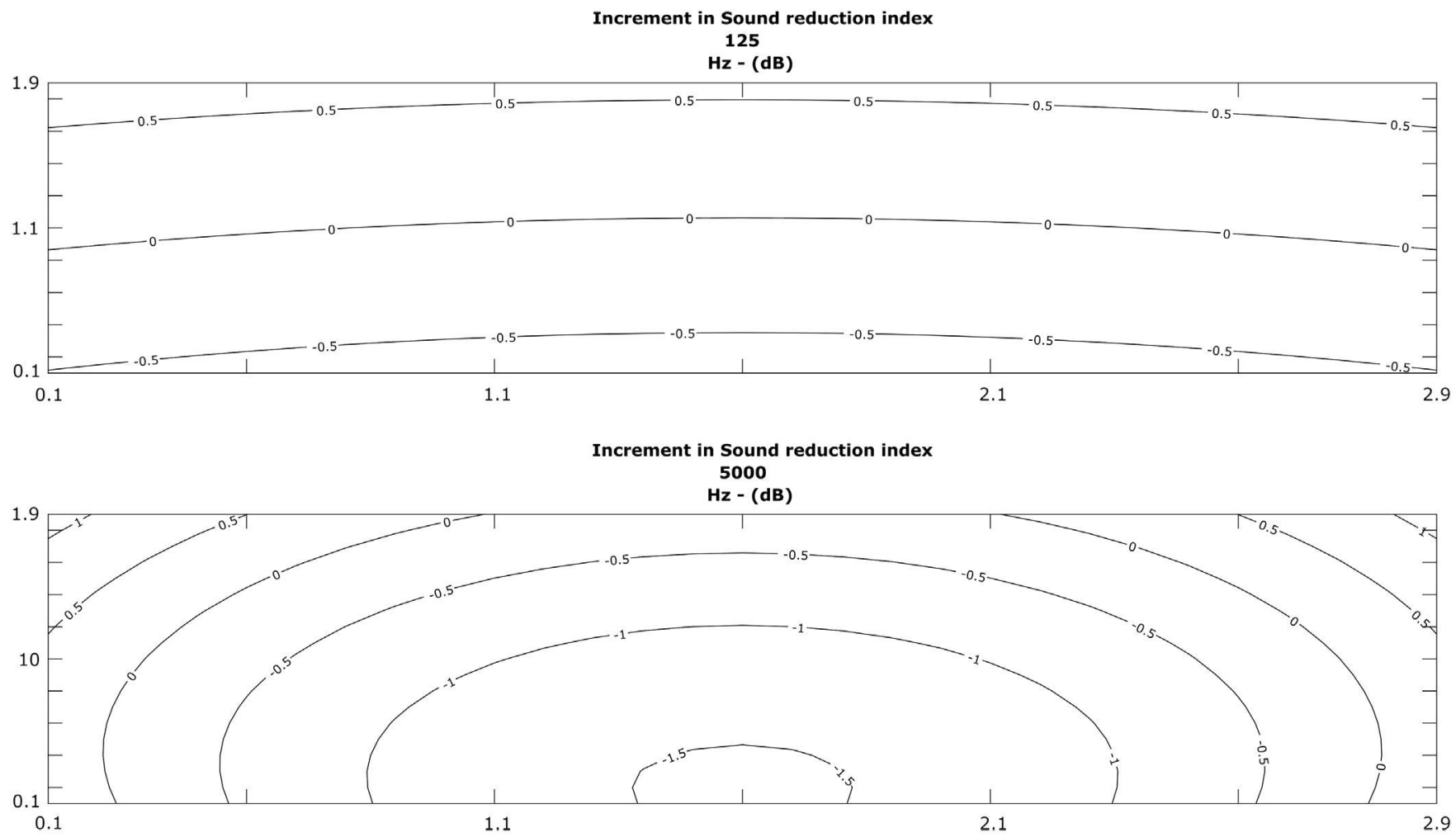

Fig. 7. Sound reduction index increments as a function of the window's location ( $x$ and $y$ mean the position of the lower-left corner of the window).

\section{Bias and variability as a function of area of the weakest element}

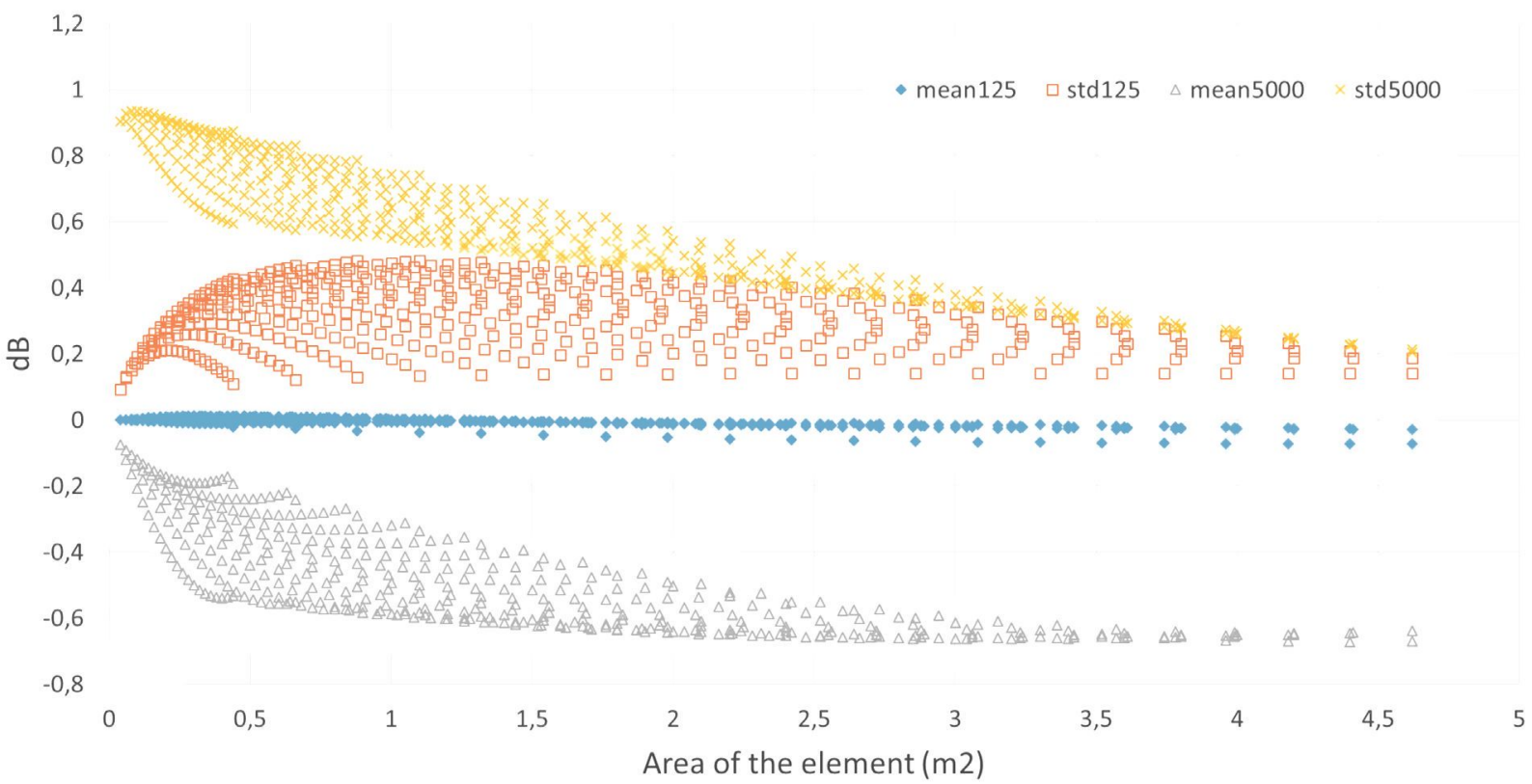

Fig. 8. Bias and variability of sound reduction index determinations as a function of the area of the window.

- When the directivity is low ( $125 \mathrm{~Hz}$ band) and the size of the element is not low (up to approximately $1-1.5 \mathrm{~m}^{2}$ ), the variability is significantly affected by the sound insulation of the weakest element. Measurements are much more sensitive to the element location when the insulation is small.

\subsubsection{Weakest element ratio}

As observed in the previous section, the ratio between the areas of the wall and the element $\left(S_{\text {wall }} / S_{\text {element }}\right)$ and the ratio between their sound reduction indexes $\left(\Delta \mathrm{R}^{\prime}=\mathrm{R}_{\text {wall }} \mathrm{R}_{\text {element }}\right)$ are factors that affect the variability and bias of the measurement method. Eq. (7) describes this sensibility as a function of $\Delta R^{\prime}$ :

$\frac{d R_{45^{\circ}}^{\prime}}{d \cdot R^{\prime}}=\frac{1}{1+\frac{S_{\text {wall }}}{S_{\text {element }}} \cdot 10^{-\frac{R^{\prime}}{10}}}$

When this coefficient is applied (analytic method) to the bias and variability calculated in Scenarios 1 and 2, Fig. 12 is obtained, which 


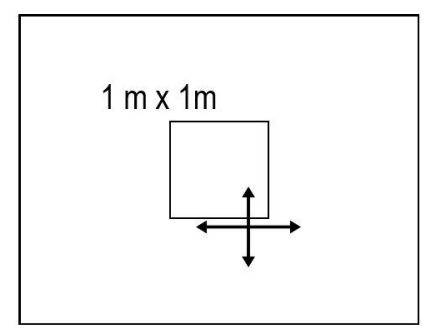

Mean $=-0.49$

Std $=0.64$

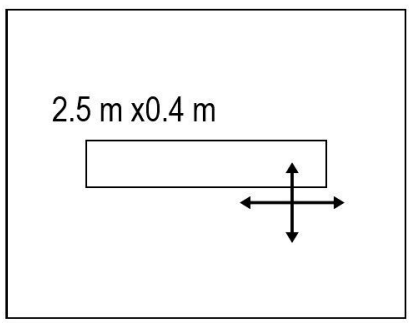

Mean $=-0.54$

Std $=0.54$

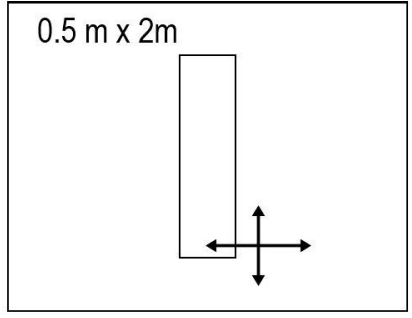

Mean $=-0.32$

Std $=0.74$

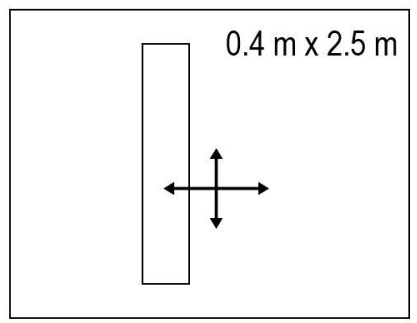

Mean $=-0.23$

Std $=0.78$

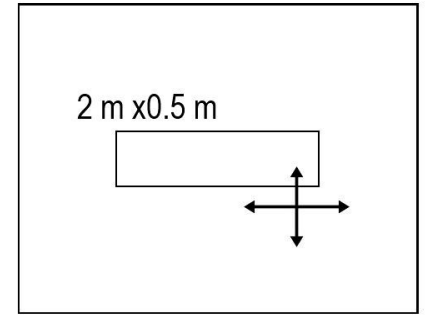

Mean $=-0.59$

Std $=0.55$

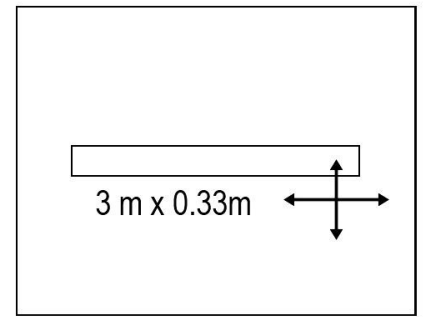

Mean $=-0.01$

Std $=0.49$

Fig. 9. Bias and variability of sound reduction index determinations with a $1 \mathrm{~m}^{2}$ area and different window shapes in a $4 \times 3 \mathrm{~m}{ }^{2}$ area wall (5000 $\mathrm{Hz}$ ).

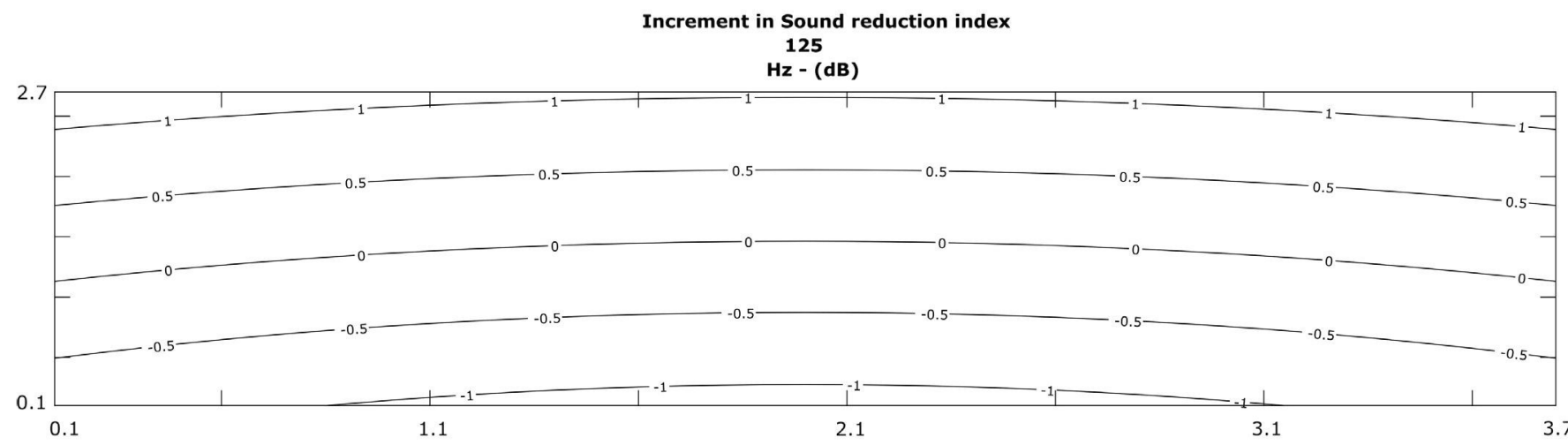

Increment in Sound reduction index

5000

$\mathrm{Hz}-(\mathrm{dB})$

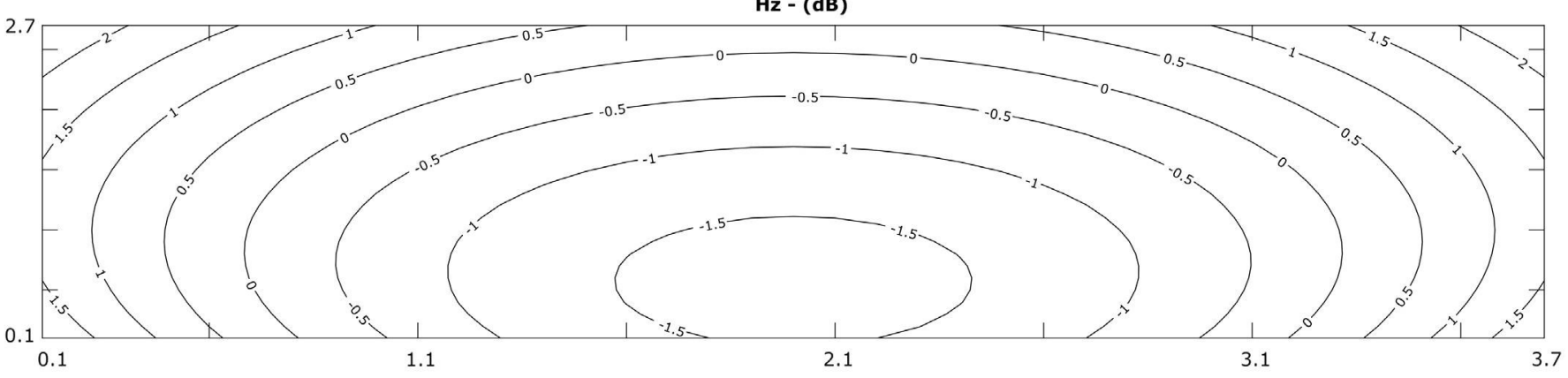

Fig. 10. Sound reduction index increments as a function of the air inlet location ( $x$ and $y$ mean the position of the lower-left corner of the air inlet).

is coincident to that obtained by applying the numeric method in previous sections. Then, Fig. 12 shows how $\Delta \mathrm{R}^{\prime}$ affects the method's bias and variability in the facades that are shown in Scenarios 1 and 2:

- The larger $\Delta \mathrm{R}^{\prime}$ and the bigger the area of the element (compared to area of the wall), the bigger the measurement method's bias (negative) is.
- The smaller the area of the element, the more important $\Delta \mathrm{R}^{\prime}$ becomes; the variability grows until $\Delta \mathrm{R}^{\prime}>40 \mathrm{~dB}$ (approximately). Although this is also true for the bias, it remains negligible in the case of small elements (bias $=-0.07 \mathrm{~dB}$ ).

- In the case of a bigger element (like the window in Scenario 1), a $\Delta \mathrm{R}^{\prime}$ value over $20 \mathrm{~dB}$ makes the bias and variability reach their maximums. 


\section{Bias and variability as a function of area of the weakest element}

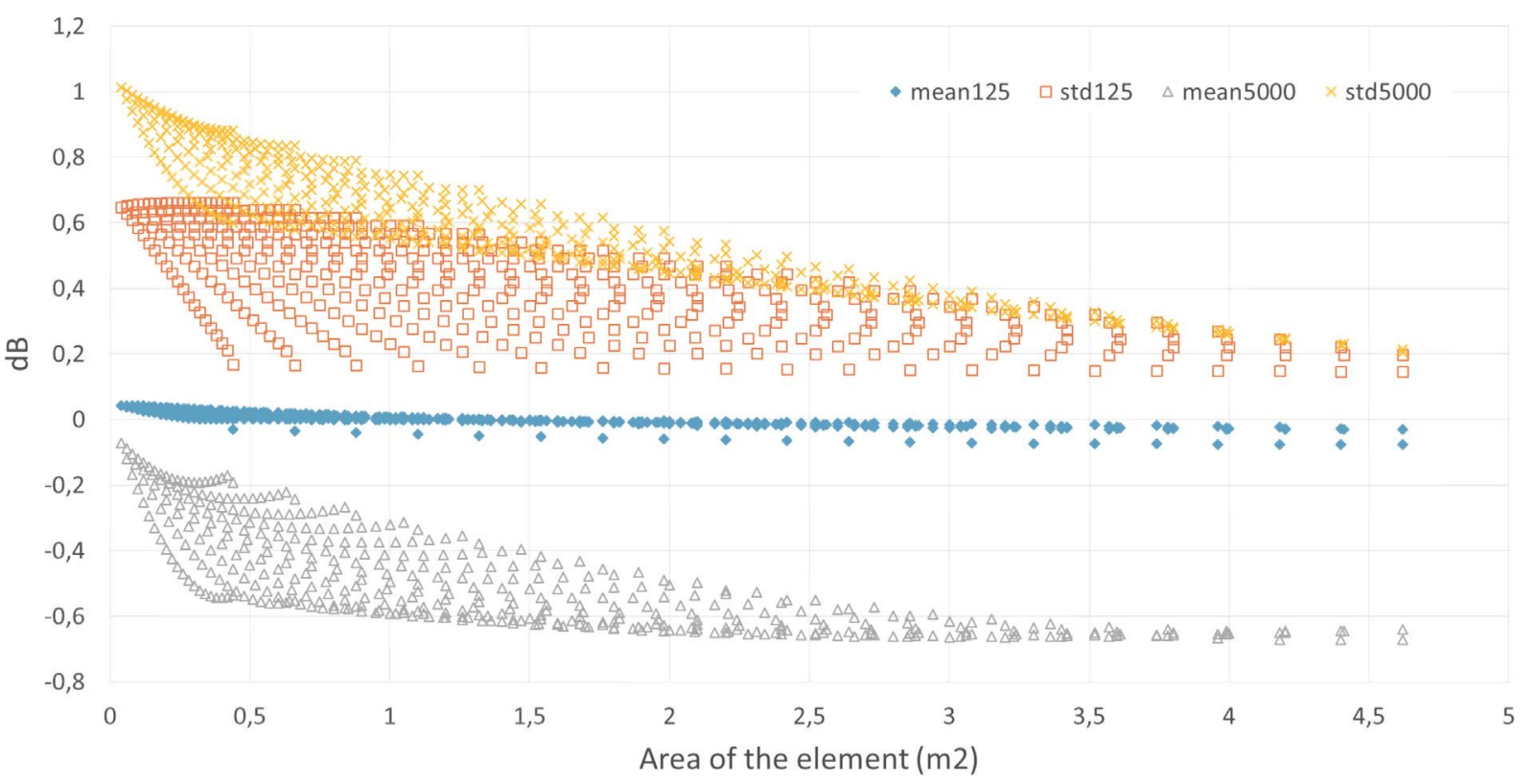

Fig. 11. Bias and variability of sound reduction index determinations as a function of the area of the air inlet.

\section{Discussion}

In view of the objectives of this research, it was decided to not investigate the influence of interferences or the angle of incidence of sound. These phenomena have been addressed in previous research [14-19], where it has been proven that they have an influence on the test results. However, even if the influence of those phenomena did not exist (or could be minimized), the factors that are derived from the directivity of the source and the location of the facade elements would modify the test results.

In this research, the sound reduction index of the facade $\left(\mathbf{R}_{45^{\circ}}\right)$ has been analyzed, and it has been observed that the sound insulation of each small part of the facade is weighted according to the combined effect of the distance and the directivity pattern of the loudspeaker. In real tests, the facade's sound insulation is usually described in terms of standardized level differences $\left(D_{2 m, n T}\right)$. For each study case, the incident sound intensity and the transmitted intensity remain as described in the previous sections. Consequently, the loudspeaker directivity will determine the outdoor sound level $\left(\mathrm{L}_{1}\right)$, which might add an additional contribution to the uncertainty as the directivity changes from loudspeaker to loudspeaker. However, if the loudspeaker does not change among observations, $\mathrm{L}_{1}$ will stay the same, and the conclusion in $\mathrm{R}_{45}^{\prime}$ can be directly applied to $\mathrm{D}_{2 \mathrm{~m}, \mathrm{nT}}$ (loudspeaker orientation errors are also outside the scope of this study). How can the variability of the method be reduced? If there is a possibility of locating the speaker at a greater distance from the front of the facade, the vari-

\section{Bias and variability as a function of sound reduction difference between weak and strong facade elements}

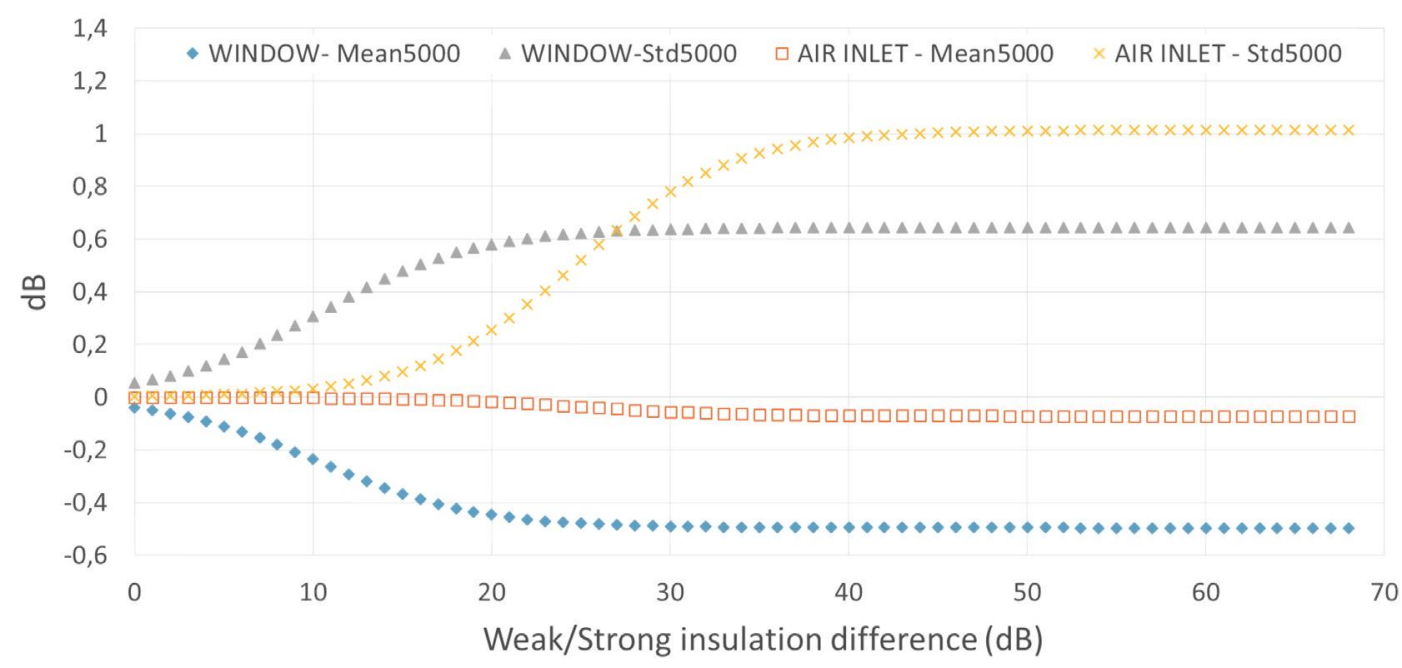

Fig. 12. Bias and variability of sound reduction index determinations as a function of the area of the air. 
ability will be lower (both the distance and directivity variabilities become smaller). Are there any other options?

If a different location was selected to determine $\mathrm{L}_{1}$, the concussions would not change as long as that new position remains fixed during the analysis. A different definition for the descriptor would be obtained, but that definition would not change over the course of the analysis; therefore, the variability would remain unchanged.

No further benefits would be obtained if additional positions were used to spatially average $\mathrm{L}_{1}$. In fact, if the practitioner could freely select these new positions, there would be a new contribution to uncertainty that derived from the position selection.

In an initial attempt to optimize the method so that its variability decreases, the following options have been tested:

- Option 1. The outdoors microphone is placed in front of the weakest element, instead of in front of the center of the facade. The loudspeaker is placed following the international standard constraints, and it points toward the facade center.

- Option 2. The outdoors microphone is placed in front of the weakest element. The loudspeaker is placed following the constraints in the international standard, but it points toward the weakest element, instead of toward the facade's center.

- Option 3. The outdoors microphone is placed in front of the weakest element. The facade's center is no longer a reference for the location of the speaker. The weakest element center is the new reference point, which will determine the location of the loudspeaker (distance, angle...).

Options 1 and 2 have some advantages, as they can address the problems derived from directivity, but they also have serious drawbacks as they cannot correct the effect derived from the facade to loudspeaker distance. A better performance can be achieved with Option 3. Option 3 seems to achieve good results in Scenarios 1 and 2 (smaller variability), while in this option, the incident sound in the weakest element remains the same no matter its location along the facade. The uneven coverage is a minor issue, as it is possible to focus the maximum sound intensity on the weakest element. A new drawback arises in this new measurement method, again deriving from the directivity of the loudspeaker: the sound intensity ratio between the weakest element and a $2-\mathrm{m}$ measurement point in front of it will not be the same for different loudspeakers and it will be a function of the frequency band. Therefore, a correction will be required. In fact, this option (and the other two) implies a new measurement method; therefore, there will be some bias that must be studied in depth, as it will imply corrections that are to be applied to $L_{1}$ determinations or new constraints to loudspeakers.

When this new method is applied (option 3 ), the variability in scenario 1 is:

$\operatorname{mean}_{125}=1.69 \mathrm{~dB} ; \quad \operatorname{mean}_{5000}=-0.58 \mathrm{~dB} ; \quad \operatorname{std}_{125}=0.09 \mathrm{~dB} ;$

$\operatorname{std}_{5000}=0.00 \mathrm{~dB}$

While in scenario 2 :

$\operatorname{mean}_{125}=1.67 \mathrm{~dB} ; \quad \operatorname{mean}_{5000}=-0.67 \mathrm{~dB} ; \quad \operatorname{std}_{125}=0.01 \mathrm{~dB} ;$ $\operatorname{std}_{5000}=0.00 \mathrm{~dB}$

When a loudspeaker with higher directivity (double radius) is used, the variability remains approximately the same, but the bias in the high frequency bands becomes very high. This is a bias that can be easily corrected with prior knowledge of the directivity of the source.

\section{Conclusions}

This paper focuses on the description of the effect that the nonuniform incident sound produces in facade sound insulation tests, depending on the location of the weakest element of the facade. The main concussions are as follows:

1. This is a phenomenon that, on its own, can have a relevant effect on facade insulation determinations. For instance, depending on the location of the air inlet in scenario 2, the results can differ by up to $4 \mathrm{~dB}$. In Scenario 1 with a $1 \mathrm{~m} \times 1 \mathrm{~m}$ window, the differences are smaller but are still high (up to $2 \mathrm{~dB}$ ). The uncertainty in these tests is often higher, but there is an additional contribution that cannot be neglected.

2 . In the low frequency range, the loudspeaker will be very omnidirectional, and the non-uniformity sound incidence is caused by the distance from the source to the receiver. Whereas in the high frequency range, the directivity effect will usually be higher than the distance effect. In this research, both cases have been treated separately, but future research must determine the combined influences in the valuation of the overall sound insulation ratings.

3. In the case where the facade consists of just one homogeneous element, the effect of a non-uniform incident sound field will be negligible. However, the weaker the weak element is, the higher the influence of the element location. In this context, weaker means higher sound transmission (lower insulation and/or bigger area). The bigger the difference between the sound insulation of the elements and the smaller the weakest element size, the more important is the location of this weakest element along the facade.

4. Not only the area of the element but also its horizontal and vertical dimensions will affect the results.

5. When the procedures in the international standards are applied, the measurement setup does not depend on the location of the facade element; consequently, the incident sound will not be dependent on the element location. However, the transmitted sound $\left(\mathrm{L}_{2}\right)$ will. Therefore, the variability of the sound insulation estimations is only derived from the variability of the transmitted sound $\left(\mathrm{L}_{2}\right)$, which mainly depends on the weakest element location for a given incident sound. It does not matter if $L_{1}$ is measured in several locations or in a different position since it does not depend on the element's location.

6. In addition to other factors (interference, angle of incidence...) and even if the coverage criterion in the standard is met, the non-uniform coverage will have an influence on the test results, which depends on the weakest element's location. This is an uncertainty contribution, which is added to others and should be minimized.

7. A new method to optimize the repeatability of the test results has been proposed, where the measurement setup is selected depending on the location of the weakest element, trying to keep constant the incident sound on that element. Although it still has to be tested in depth (simulations) and validated in the field (real measurements), the primary results are very promising. Future research on this proposal should pay attention to the influence that the lack of precision during the orientation of the loudspeaker could have on the results. Further constraints should be implemented as in certain scenarios, it might be difficult for the practitioner to determine which is the weakest element prior to testing. In those cases, the variability problem would remain similar to that in the standardized method.

\section{References}

[1] WHO.R.o.f. Europe. Burden of disease from environmental noise: quantification of healthy life years lost in Europe. World Health Organization; 2011. 
[2] Rasmussen B, Rindel JH. Sound insulation between dwellings - descriptors applied in building regulations in Europe. Appl Acoust 2010;71:171-80.

[3] Rasmussen B. Sound insulation between dwellings - requirements in building regulations in Europe. Appl Acoust 2010;71:373-85.

[4] Asensio C, Pavón I, Recuero M, Ausejo M. Airport noise insulation programs: the Spanish case. Noise Vib Worldwide 2012;43:8-15.

[5] ISO. ISO 16283-3:2016. Acoustics - field measurement of sound insulation in buildings and of building elements - part 3: facade sound insulation; 2016.

[6] Sánchez Bote JL, Pedrero González A, Gómez Alfageme JJ. Influence of loudspeaker directivity and measurement geometry on direct acoustic levels over facades for acoustic insulation tests with the International Standard ISO 140-5. Appl Acoust 2012;73:440-53.

[7] Sănchez Bote JL, Pedrero González A, Gómez Alfageme JJ. Procedure for verification of sound source coverage over facades according to the International Standard ISO 140-5. Appl Acoust 2012;73:977-85.

[8] Sánchez Bote JL, Gómez Alfageme JJ. Procedimiento para la medición y verificación de la directividad y cobertura de una fuente sobre un elemento de fachada de acuerdo a la norma ISO 140-5; 2011.

[9] Pedrero Gonzălez A, Sánchez Bote Jl, Ulin Nabatov V, Díaz Sanchicriân C Influence of loudspeaker directivity on the measurement uncertainty of the acoustic testing of facades; 2011.

[10] Navacerrada MA, Pedrero A, Díaz C. Study of the uncertainty of facade sound insulation measurements: Analysis of the ISO 12999-1 uncertainty proposal Appl Acoust 2016;114:1-9.
[11] ISO. ISO 140-5:1998. Acoustics - measurement of sound insulation in buildings and of building elements - part 5: field measurements of airborne sound insulation of facade elements and facades; 1998.

[12] ISO. ISO 9613-2:1996. Attenuation of sound during propagation outdoors. General method of calculation; 1996.

[13] EN, EN 12354-3:2000. Building acoustics. Estimation of acoustic performance in buildings from the performance of elements. Airborne sound insultation against outdoor sound; 2000.

[14] Berardi U. The position of the instruments for the sound insulation measurement of building facades: from ISO 140-5 to 1 SO 16283-3. Noise Control Eng J 2013;61:70-80.

[15] Hall FL, Papakyriakou MJ, Quirt JD. Comparison of outdoor microphone locations for measuring sound insulation of building facades. J Sound Vib $1984 ; 92: 559-67$.

[16] Olafsen S, Bard D, Strand MK, Espejo TF. Methods of field measurements of facade sound insulation. Noise Control Eng J 2015;63:467-77.

[17] Hopkins C. Revision of international standards on field measurements of airborne, impact and facade sound insulation to form the ISO 16283 series. Build Environ 2015;92:703-12.

[18] ASTM International. ASTM E966-10e1, standard guide for field measurements of airborne sound insulation of building facades and facade elements; 2010.

[19] Berardi U. Comparison among methods to measure the sound insulation of building facades; 2011. 